\title{
Der Arbeitskreis Biologische und Ökologische Prozesse
}

\section{Heike Link}

Department Maritime Systeme, Universität Rostock, 18059 Rostock, Deutschland

Institut für Biowissenschaften, Universität Rostock, 18059 Rostock, Deutschland

Correspondence: Heike Link (heike.link@uni-rostock.de)

Published: 20 May 2021

\section{Der Arbeitskreis „Biologische und Ökologische Prozesse" in der Entwicklung}

Ein aktiver Arbeitskreis Biologie - das ist auch nach 10 weiteren Jahren der Deutschen Gesellschaft für Polarforschung ein Entwicklungsfeld mit Potential.

Mit viel Elan wurde der Arbeitskreis mit dem Titel „Biologische und Ökologische Prozesse" 2015 neu belebt, und in zwei folgenden Treffen wurden konkrete Pläne und Bedarfe erarbeitet. Ein Mehrwert aus der Zusammenarbeit im AK boten zu diesem Zeitpunkt für die Teilnehmer Themen an den Schnittstellen zwischen Land und Meer sowie Pflanze und Tier. Die geplanten Workshops zur Methoden-basierten Synthese von Ergebnissen aus unterschiedlichen Fachrichtungen konnten jedoch leider nicht realisiert werden. Seitdem ist der AK „Bio- Öko“ kaum aktiv, was jedoch keinesfalls für die Biologen in der Polarforschung in Deutschland an sich gilt. Insbesondere international engagieren sich deutsche Polarbiologen stark in fachübergreifenden Forschungsprogrammen. Als Beispiel aus den letzten zehn Jahren seien die erfolgreichen Arbeiten aus dem AnT-ERA- (Antarctic Thresholds - Ecosystem Resilience and Adaptation, zuletzt Gutt et al., 2021) und dem AntEco-Programm (State of the Antarctic Ecosystem, zuletzt Ingels et al., 2021) sowie der Remote Sensing Action Group in SCAR genannt.

Das Potential dieses Arbeitskreises der DGP liegt in der Vernetzung über Institutionen hinweg, um so bisher getrennte Themenbereiche neu zu beleuchten. Nach wie vor sind Fragestellungen zur Integration von terrestrischen und marinen Funktionsweisen in der polaren Ökologie kaum beleuchtet. Aber auch die kombinierte Nutzung neuer und traditioneller Methoden in der Beschreibung von Lebensgemeinschaften in Arktis und Antarktis könnte durch Impulse aus dem AK „Biologische und Ökologische Prozesse“ vorangetrieben werden.

\section{Interview mit Dr. Heike Link, Sprecherin des AK} Biologische und Ökologische Prozesse

APECS Germany: Kurz zu Ihrer Person und Ihrem beruflichen Werdegang. Wollten Sie schon immer Polarforscherin werden? Wann und warum haben Sie sich für die Polarforschung entschieden?

H. Link: „Schon immer“ ist ein sehr weit gefasster Begriff. Sicher war für mich, dass ich mich mit wirbellosen marinen Tieren beschäftigen wollte. Endgültig für die Polargebiete hat mich dabei erst ein Vortrag von Julian Gutt im Studium in Heidelberg fasziniert. Das besondere Zusammenspiel von Eis, Primärproduktion und den einzigartigen Tiergemeinschaften am Meeresboden gibt uns noch viele Fragen auf. Und das in einer noch kaum anthropogen gestörten Umwelt zu untersuchen verspricht doch wissenschaftlich spannende Erkenntnisse für die Ökologie allgemein! Während meiner Doktorarbeit in der kanadischen Arktis wurde mir dann klar, dass die Polargebiete bald deutlich verändert, genutzt und gestört werden. Deshalb finde ich es wichtig, mit meiner Wissenschaft zum besseren Verständnis der Polargebiete beizutragen, das letztendlich auch in die zukünftigen Entscheidungen zu Nutzungen eingehen kann. Hoffentlich!

APECS Germany: Warum setzen Sie sich für das Thema Ihres AK ein? Was macht dieses Forschungsfeld für Sie so besonders, dass Sie dafür so viel Zeit und Einsatz bringen?

H. Link: Eigentlich müsste ich noch viel mehr Zeit einsetzen, im Moment läuft der AK eher auf Sparflamme.

Das Forschungsfeld ist besonders für mich, da es die Möglichkeit gibt fachübergreifend zu arbeiten, sowie neue Fragen zu entwickeln. Zum Beispiel könnten das Gemeinsamkeiten aus dem terrestrischen und marinem Feld sein oder Forschung an der Schnittstelle. Außerdem würde ich gern Ökologie und Würmer-zähler auch für Modellierer interessant 
machen. Außerdem möchte ich Herausforderungen der Feldarbeit durch abgestimmte Aktivitäten erleichtern.

APECS Germany: Was gefällt Ihnen an Ihren Aufgaben als AK Leiter ganz besonders? Hat sich dadurch Ihre Sicht auf etwas geändert?

H. Link: Ich freue mich immer sehr, wenn ich Wissenschaftler, die vorher noch nie gemeinsam über eine Forschungsfrage nachgedacht haben, zusammenbringen kann. Da sprudelt es manchmal nur so vor Ideen. Ein bisschen wurde ich aber auch desillusioniert über die Umsetzung von Ideen. Schwierig ist hier, dass die etablierten Wissenschaftler meist wenig Zeit oder Priorität dafür einbringen können, und jungen Wissenschaftler die notwendige langfristige Verortung oder Perspektive fehlt.

Auch kommt es mir so vor, als ob es unter Biologen oft leichter ist international zusammenzuarbeiten als national. Vor allem die AWI-Struktur für biologische Polarforschung deckt in Deutschland schon sehr viel ab. Daher ist es nicht einfach, eine kritische Masse an Polarbiologen aus Universitäten UND anderen Instituten zusammenzubringen.

APECS Germany: Wenn Sie in die Zukunft schauen, was würden Sie gerne im Feld Ihres Arbeitskreises in fünf Jahren herausgefunden haben? Was ist in Ihren Augen die dringendste Frage im Feld Ihres Arbeitskreises?

H. Link: In der Polarforschung sind noch viele Fragen offen. Wir wissen ja z.B. noch gar nicht, wie viele Arten es wirklich in den Polargebieten gibt, wie viele wir durch den Klimawandel verlieren werden, und was dieser Verlust für die polaren Ökosysteme bedeuten wird. Das gilt an Land und im Meer, von Diversität bis zu Stoffflüssen, und aus biologischer Sicht wissen wir noch weniger, wie diese beiden Systeme interagieren. Meiner Meinung nach werden wir in den nächsten Jahren mehr über traditionelle Strukturen (marin-terrestrisch, biologisch-geologisch, taxonomischmolekularbiologisch) hinweg arbeiten und so neue Erkenntnisse produzieren.

Wichtig ist auch die Ideenentwicklung. Es braucht den Mix aus erfahrenen PIs und Nachwuchs. Sehr junge Wissenschaftler am Anfang ihrer Karriere sind sehr motiviert, ihnen fehlt aber oft noch die nötige Erfahrung.

APECS Germany: Was darf bei der Arbeit im Feld für Sie nie fehlen?

H. Link: Guter Kaffee! Als Benthosforscherin muss ich viel nachts arbeiten, und sowieso arbeiten wir im Feld oft bis zum Umfallen. Das Aroma eines guten Kaffees kann die Stimmung fast so viel erhellen wie ein gut gelauntes eingespieltes Team.

APECS Germany: Was war Ihre einprägsamste (lustig/beängstigend/seltsam) Erfahrung in der Polarforschung die Sie gemacht haben?

H. Link: Da gibt es so viele! Unvergesslich unheimlich war mein erster Schritt aufs Eis mit hunderten Metern Meer unter mir, damals von der CCGS Amundsen in Kanada aus. Ebenso wenig werde ich den Blick und die Begeisterung meines ersten Masterstudenten vergessen, als er das erste
Mal in Troms $\emptyset$ an Bord von Polarstern ging. Es ist toll so etwas jungen Menschen über die Lehre ermöglichen zu können.

Eine andere sehr einprägsame Erfahrung war, als ich zum ersten Mal die Benthosfauna der Antarktis gesehen habe. Vorher war ich sehr lang auf die Arktis fokussiert, aber das Benthal dort ist was ganz anders. Natürlich ist der antarktische Ozean viel älter und heterogener und hat dadurch eine ganz andere Fauna. Als letztes kann ich noch ein Erlebnis nennen: 44h Arbeit auf See am Stück! Als ich wie in Trance Halluzinationen bekam, wusste ich, dass Schlaf auch eine Frage der Sicherheit ist.

APECS Germany: Als ECR (Early Career Researcher) interessiert mich besonders, ob Sie mit besonderen Schwierigkeiten konfrontiert wurden beim Start Ihrer Karriere. Was würden Sie mir raten, wenn Sie nur einen Tipp geben könnten?

H. Link: Mut haben und nicht verlieren. Mut, nein zu sagen - z.B. zu einer Doktorarbeit, die einen nicht überzeugt, auch wenn man noch keine andere hat. Mut, weiterzumachen, auch wenn man viel Kritik eingesteckt hat. Mut, die eigene Forschung allen zu vermitteln. Mut, alte Strukturen oder die Heimat zu verlassen und neu anzufangen.

Auch kann es nie schaden die mögliche Arbeitsstellen bzw Institute auf deren langfristige Perspektive zu untersuchen. Immer wieder Mut haben kostet viel Energie.

APECS Germany: Und zum Abschluss noch eine letzte aktuelle Frage: wie hat COVID-19 das wissenschaftliche Arbeiten verändert?

H. Link: Für die praktische Arbeit gibt es natürlich mehr Organisationsaufwand, zum Beispiel um Laborarbeiten zu koordinieren. Das verschiebt sich jetzt alles um ein paar Monate. Da ich auch für die Koordination des Forschungsschwerpunktes an der Uni Rostock zuständig bin, hört auch dort die Arbeit nicht auf. Es kommen viele Fragen auf, wie zum Beispiel Netzwerkkommunikation und Workshops neu organisiert werden.

Das Ungleichgewicht zwischen Wissenschaftlern mit und ohne Familie hat sich vergrößert, und dass kann insbesondere für die befristeten Nachwuchswissenschaftler Konsequenzen haben. In diesen „beruhigten“ Zeiten konzentrieren sich die etablierten und Wissenschaftler ohne Kinder mehr auf Publikationen und Anträge im Gegensatz zu anderen. Ich höre da oft den Satz ,Jetzt haben wir ja Zeit für ...“. Aber diese Zeit ist nicht bei allen gleich vorhanden.

Das Gespräch führte Charlotte Haugk im Mai 2020. 


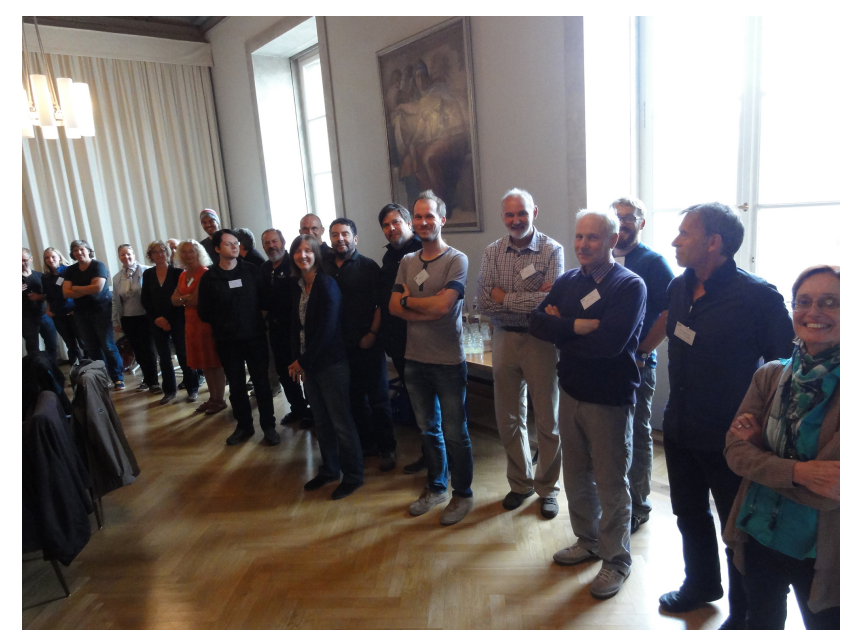

Abb. 1. „Neugründung“ des Arbeitskreises Biologische und Ökologische Prozesse während der Internationalen Polartagung 2015 in München (Foto: Heike Link).

Datenverfügbarkeit. Für diesen Artikel wurden keine Datensätze genutzt.

Interessenkonflikt. Die Autor*innen erklären, dass kein Interessenkonflikt besteht.

Begutachtung. This paper was edited by Donovan Dennis.

\section{Literatur}

Gutt, J., Isla, E., Xavier, J. C., Adams, B. J., Ahn, I.-Y., Cheng, C.-H. C., Colesie, C., Cummings, V. J., di Prisco, G., Griffiths, H., Hawes, I., Hogg, I., McIntyre, T., Meiners, K. M., Pearce, D. A., Peck, L., Piepenburg, D., Reisinger, R. R., Saba, G. K., Schloss, I. R., Signori, C. N., Smith, C. R., Vacchi, M., Verde, C., and Wall, D. H.: Antarctic ecosystems in transition - life between stresses and opportunities, Biol. Rev., https://doi.org/10.1111/brv.12679, 2021.

Ingels, J., Aronson, R. B., Smith, C. R., Baco, A., Bik, H. M., Blake, J. A., Brandt, A., Cape, M., Demaster, D., Dolan, E., Domack, E., Fire, S., Geisz, H., Gigliotti, M., Griffiths, H., Halanych, K. M., Havermans, C., Huettmann, F., Ishman, S., Kranz, S. A., Leventer, A., Mahon, A. R., McClintock, J., McCormick, M. L., Mitchell, B. G., Murray, A. E., Peck, L., Rogers, A., Shoplock, B., Smith, K. E., Steffel, B., Stukel, M. R., Sweetman, A. K., Taylor, M., Thurber, A. R., Truffer, M., van de Putte, A., Vanreusel, A., and Zamora-Duran, M. A.: Antarctic ecosystem responses following ice-shelf collapse and iceberg calving: Science review and future research, WIREs Clim. Change, 12, e682, https://doi.org/10.1002/wcc.682, 2021. 